\title{
A Longitudinal Study of Industrial and Clerical Workers: Predictors of Upper Extremity Tendonitis
}

\author{
Robert A. Werner, ${ }^{1,2,3,4,6}$ Alfred Franzblau, ${ }^{3,4,5}$ Nancy Gell, ${ }^{1,3}$ \\ Sheryl S. Ulin, ${ }^{4}$ and Thomas J. Armstrong ${ }^{3,4}$
}

\begin{abstract}
Upper extremity tendonitis (UET) associated with work activity is common but the true incidence and risk factors can best be determined by a prospective cohort study. This study followed a cohort of 501 active workers for an average of 5.4 years. Incident cases were defined as workers who were asymptomatic at baseline testing and had no prior history of UET and went on to be diagnosed with an UET during the follow-up period or at the followup evaluation. The incident cases were compared to the subset of the cohort who also had no history of an UET and did not develop tendonitis during the study. The cumulative incidence in this cohort was $24.3 \%$ or $4.5 \%$ annually. The factors found to have the highest predictive value for identifying a person who is likely to develop an UET in the near future included age over 40, a BMI over 30, a complaint at baseline of a shoulder or neck discomfort, a history of CTS and a job with a higher shoulder posture rating. The risk profile identifies both ergonomic and personal health factors as risks and both categories of factors may be amenable to prevention strategies.
\end{abstract}

KEY WORDS: cumulative trauma disorders; musculoskeletal diseases; tendonitis; occupational diseases.

\section{INTRODUCTION}

Upper extremity musculoskeletal disorders (UEMSD) include a variety of musculoskeletal and nerve disorders that can be related to overuse of the upper limb. There has been a tremendous increase in the numbers of reported cases of UEMSDs in the last two decades (1,2). Data reported in the Bureau of Labor Statistics from 1994 demonstrate an incidence rate of $1.0-21 \%$ per year in high risk occupations such as clothing manufacturing, meat packing plants and auto assembly plants (3-5). The reports of incidence and

\footnotetext{
${ }^{1}$ Department of Physical Medicine and Rehabilitation, University of Michigan Health System, Ann Arbor, Michigan.

${ }^{2}$ Veteran Affairs Medical Center, Ann Arbor, Michigan.

${ }^{3}$ Department of Environmental Health Sciences, School of Public Health, University of Michigan, Ann Arbor, Michigan.

${ }^{4}$ Center for Ergonomics, Industrial and Operations Engineering, University of Michigan, Ann Arbor, Michigan.

${ }^{5}$ Department of Emergency Medicine, University of Michigan Health System, Ann Arbor, Michigan.

${ }^{6}$ Correspondence should be addressed at Department of Physical Medicine and Rehabilitation, University of Michigan Health System, Ann Arbor, Michigan; e-mail: rawerner@med.umich.edu.
} 
prevalence in the literature depend upon the case definition. Researchers who collect symptom and physical examination data directly from workers as an active surveillance program report higher numbers compared to those reports compiled from workers compensation claims or passive surveillance programs. Among the eight epidemiologic studies reviewed by National Institute for Occupational Safety and Health (NIOSH) for the relationship of hand/wrist tenosynovitis and work activity, the prevalence or incidence rates of hand/wrist tenosynovitis ranged from 4-56\% among exposed workers and 0-14\% among unexposed groups (3). The prevalence rate ratio (PRR) of tenosynovitis of the hand/wrist among meatcutters was 3.1 (6). McCormack et al. reported a PRR among production textile workers of 2.0 (7). Among the 20 epidemiologic studies that evaluated the relationship between shoulder musculoskeletal disorders and workplace factors, there is evidence of a positive association between highly repetitive work and shoulder disorders (3). Only three studies specifically addressed the health outcome of shoulder tendonitis and these evaluated the combined exposure of high repetition and awkward postures or static shoulder loads (3). The incidence rates for shoulder tendonitis among high risk occupations is estimated at 15-20\% with a PRR estimated at 2-6 times that of low exposure occupations (8). The PRR for elbow tendonitis was 1.2-3.5 for high exposure occupations (3).

Upper extremity tendonitis (UET) is typically localized at the shoulder, elbow, wrist or hand. The subject complains of localized pain that is reproduced with palpation of the tendon or by activating the muscle that attaches to the tendon. In advanced cases there can be visible swelling and/or erythema along the involved tendon sheath. Common types of tendonitis in the UE include rotator cuff strain (shoulder), lateral and medial epicondylitis (elbow) and DeQuervain's tenosynovitis (wrist).

The ergonomic risk factors associated with UEMSDs include repetitiveness of work, forceful exertions, mechanical stress, posture, temperature, and vibration (9). These risk factors are present for many industrial and clerical jobs and the review by NIOSH found strong epidemiologic evidence in support of these risk factors (3). The risk is highest when there is a combination, especially high force and hand repetition. Exposure to vibrating tools is considered an independent risk factor.

\section{METHODS}

This was a longitudinal study of workers from four industrial and three clerical work sites. Of the 985 subjects who participated in a baseline study, $501(51 \%)$ were screened an average of 5.4 years later. Excluding subjects that could not be contacted, there was a $74 \%$ participation rate at follow-up. Eighteen percent $(n=179)$ declined to participate and $31 \%(n=305)$ of the original 985 could not be contacted. Subjects were eligible to participate if they were in the same job, had changed jobs, or retired. All subjects underwent a directed physical examination of the upper extremities and completed a symptom questionnaire. Electrodiagnostic testing of the median and ulnar sensory nerves was also performed according to the techniques described by Kimura (10). All jobs were assessed and rated for ergonomic exposures at baseline (11). Each job was rated according to the American Congress of Governmental Industrial Hygienists' (ACGIH) threshold limit values (TLV) for hand activity level based upon the hand repetition level and the normalized peak force (12). Psychosocial variables were assessed using a questionnaire based on the one developed by Karasek (13). The areas assessed included estimates of skill discretion, 
job insecurity, perceived stress and job satisfaction based on the decision latitude of the worker and the psychological demands placed upon the worker. Each worker was weighed and measured for height to calculate the body mass index (BMI, $\left.\mathrm{kg} / \mathrm{m}^{2}\right)$.

Workers at baseline who did not have any UET but went on to be diagnosed with a regional tendonitis of the upper extremity were identified. If a worker did not report a physician diagnosis of tendonitis prior to the initial evaluation AND the physical examination and symptoms reported did not establish a current diagnosis of tendonitis, these workers were labeled as 'No UET' at baseline. If these workers did not report a physician who diagnosed tendonitis during the study evaluation, and the final physical examination and symptoms reported did not establish a current diagnosis of tendonitis, these workers were labeled as 'No UET' at the final evaluation. The diagnosis of an incident case of UET was established in two ways. Either the worker reported that a physician had diagnosed them with an UET, or based upon their symptom reporting, in combination with the physical examination, we established a diagnosis of current UET at the follow-up screening. A report of symptoms of pain, stiffness, aching, burning or tenderness that had been present for a week or more or occurred on three or more episodes in combination with an associated physical finding of pain with palpation or with resistive movements at the wrist, elbow or shoulder established the current diagnosis of tendonitis (14). If a worker in the 'No UET' at baseline reported a new diagnosis of tendonitis during the study period, they were considered an 'Incident Case' of UET.

\section{STATISTICS}

We evaluated which demographic, ergonomic, psychosocial variables and symptom complaints at baseline would be predictive of Incident Cases. The subset of the longitudinal study cohort identified with 'No UET' at baseline was selected for analysis. The dependent variable was whether these workers developed into incident cases. The independent variables included demographic variables, i.e., age, gender, medical history, obesity, smoking history, and exercise levels as well as all ergonomic posture and force variables and the psychosocial work-related variables of skill discretion decision authority, coworker support, job insecurity, job satisfaction and perceived stress. Electrophysiologic variables were also included as independent variables since a history of CTS is related to a higher prevalence of tendonitis (15). Initially, a univariate analysis was done and this was followed by logistic regression modeling to determine the most predictive model for identifying incident cases from the baseline data. A secondary analysis was run to determine if changes in the job might have also influenced incident cases. The analysis was first run for regional areas, elbow/wrist/hand or shoulder, and then for the upper extremity as a whole. The shoulder, elbow, wrist and hand were then combined since the incident cases for any one region was too low to run an analysis.

\section{RESULTS}

A total of 350 subjects were identified as having 'No UET' at baseline. Of these individuals, a total of 94 subjects were found to have developed an UET during the study period. This included 78 subjects identified by self report, i.e., they did not report a history of tendonitis at baseline, their physical examination at baseline was not consistent with 
tendonitis and they subsequently reported that a physician had diagnosed them as having an UET at follow-up. Additionally, 46 subjects met criteria for diagnosis of an UET at follow-up based upon their symptoms and physical examination findings. Within this group, 30 subjects already been identified by self report of a physician diagnosis of an UET.

The baseline characteristics of the subjects with 'No UET' and the incident cases of UET are presented in Table I. The groups are similar in age, gender, medical history, hand repetition level, reported exercise level, smoking history, and psychosocial variables. There was no difference between incident cases identified by our examination and those identified by the workers' personal physicians. Workers who developed an UET were more obese (BMI of 29.6 vs. 27.7, $p=0.018$ ). They were more likely to have reported hand/wrist and shoulder discomfort at the baseline screening (hand/wrist $58.5 \%$ vs. $43.2 \%, p=0.011$;

Table I. Comparison of 'Incident Cases of UET' to 'No UET'

\begin{tabular}{|c|c|c|c|}
\hline & Incident UET (n) & No UET ever (n) & $p$ \\
\hline BMI & $29.5(98)$ & $27.7(258)$ & 0.03 \\
\hline Age & $38.5(98)$ & $37.9(264)$ & 0.65 \\
\hline Gender (female \%) & $72.5 \%(98)$ & $68.6 \%(264)$ & 0.47 \\
\hline Repetition & $5.8(93)$ & $5.6(257)$ & 0.29 \\
\hline Wrist/hand/finger symptom & $60.2 \%(98)$ & $42.4 \%(264)$ & 0.00 \\
\hline Neck/shoulder symptoms & $60.2 \%(98)$ & $37.9 \%(264)$ & 0.00 \\
\hline Elbow/forearm symptoms & $28.6 \%(98)$ & $18.9 \%(264)$ & 0.05 \\
\hline All upper extremity symptoms & $78.6 \%(98)$ & $59.5 \%(264)$ & 0.00 \\
\hline Diabetes & $2.04 \%(98)$ & $1.9 \%(264)$ & 0.90 \\
\hline Rheumatoid arthritis & $2.06 \%(97)$ & $1.9 \%(264)$ & 0.90 \\
\hline Retire & $8.16 \%(94)$ & $10.6 \%(264)$ & 0.50 \\
\hline Job change & $64.2 \%(94)$ & $58.5 \%(264)$ & 0.30 \\
\hline Exercise & $61.5 \%(52)$ & $59.3 \%(150)$ & 0.78 \\
\hline Smoke & $34.6 \%(94)$ & $39 \%(266)$ & 0.45 \\
\hline Worst discomfort rating among three regions & $3.20(77)$ & $2.05(156)$ & 0.00 \\
\hline Worst discomfort rating among three regions in last 30 days & $4.23(64)$ & $3.59(117)$ & 0.20 \\
\hline Skill discretion & $24.2(94)$ & $25.5(256)$ & 0.13 \\
\hline Decision authority & $24.4(96)$ & $26.3(262)$ & 0.06 \\
\hline Created & $6.6(95)$ & $6.9(260)$ & 0.29 \\
\hline Coworker support & $11.6(68)$ & $11.7(186)$ & 0.63 \\
\hline Supervisor support & $11(93)$ & $11.5(247)$ & 0.09 \\
\hline Job insecurity & $5.3(71)$ & $5.2(190)$ & 0.64 \\
\hline Job satisfaction & $0.34(98)$ & $0.30(256)$ & 0.06 \\
\hline Perceived stress & $23.57(70)$ & $22.9(189)$ & 0.58 \\
\hline Clerical \% & $68.4 \%(98)$ & $61.4 \%(264)$ & 0.22 \\
\hline Median mononeuropathy $\geq 0.5 \mathrm{~ms}$ & $25.1 \%(98)$ & $19.2 \%(264)$ & 0.19 \\
\hline Abnormal hand diagram & $29.5 \%(98)$ & $20.7 \%(264)$ & 0.08 \\
\hline Abnormal hand activity threshold limit value (TLV) & $40.4 \%(21)$ & $37.9 \%(55)$ & 0.75 \\
\hline Median ulnar peak latency difference (dominant side) & $0.24(97)$ & $0.21(264)$ & 0.6 \\
\hline Peak force (dominant hand) & $2.89(97)$ & $2.94(256)$ & 0.66 \\
\hline New CTS at T2* or in-between & $6.12 \%(6)$ & $7.9 \%(21)$ & 0.55 \\
\hline No CTS ever & $75.5 \%(74)$ & $85.6 \%(226)$ & 0.02 \\
\hline New shoulder tendonitis at T2 or in between & $38.8 \%(38)$ & $0 \%$ & 0.00 \\
\hline No shoulder tendonitis ever & $56.12 \%(55)$ & $100 \%(264)$ & 0.00 \\
\hline New wrist/hand/finger tendonitis at T2 or in-between & $62.2 \%(61)$ & $0 \%$ & 0.00 \\
\hline No wrist/hand/finger tendonitis ever & $36.7 \%(36)$ & $100 \%(264)$ & 0.00 \\
\hline New elbow/forearm tendonitis at $\mathrm{T} 2$ or in-between & $63.3 \%(62)$ & $0 \%$ & 0.00 \\
\hline No elbow/forearm tendonitis ever & $35.7 \%(35)$ & $100 \%(264)$ & 0.00 \\
\hline CTS at $\mathrm{T} 1^{* *}$ & $11.2 \%(11)$ & $3.4 \%(9)$ & 0.00 \\
\hline
\end{tabular}

*T2-Time 2 (follow-up); ${ }^{* *}$ T1-Time one-(baseline). 
shoulder $58.5 \%$ vs. $38.7 \%, p=0.001$ ); there was a trend for more discomfort reported at the elbow as well $(27.7 \%$ vs. $19.2 \%, p=0.085)$.

Incident cases of UET tended to have hand symptoms at baseline that were consistent with carpal tunnel syndrome (CTS), i.e., tingling, burning, numbness or pain in the median nerve distribution (based upon an abnormal hand diagram score), 30.0\% vs. $20.7 \%$, $p=0.07$. The incident cases were more likely to have been diagnosed with CTS at baseline assessment (based upon an abnormal hand diagram plus confirmation by nerve conduction studies), $10.6 \%$ vs. $3.4 \%, p=0.007$. The incident cases were likely to have been diagnosed (by a physician) with CTS prior to the baseline assessment, $8.5 \%$ vs. $3.8 \%, p=0.07$.

The regional tendonitis at the shoulder, as well as the elbow/wrist/hand, demonstrated similar trends as noted in the total incident UET cases. The characteristics of these cohorts are presented in Tables II and III. For the elbow/wrist/hand analysis, there were a total of 377 subjects with 78 being defined as incident cases. The independent variables that were significant in the univariate analysis included an initial presentation with hand, wrist

Table II. Comparison of 'Incident Cases of Elbow Wrist Hand Tendonitis' to 'No Elbow Wrist Hand Tendonitis'

\begin{tabular}{|c|c|c|c|}
\hline & $\begin{array}{l}\text { Incident E/F } \\
\text { tendonitis, (n) }\end{array}$ & $\begin{array}{c}\text { No E/F tendonitis } \\
\text { ever, (n) }\end{array}$ & $p$ \\
\hline BMI & $29(69)$ & $28(314)$ & 0.28 \\
\hline Age & $38.7(69)$ & $38.2(320)$ & 0.71 \\
\hline Gender (female \%) & $71 \%(69)$ & $69 \%(320)$ & 0.71 \\
\hline Repetition & $5.5(68)$ & $5.6(313)$ & 0.74 \\
\hline Wrist/hand/finger symptom & $60.9 \%(69)$ & $47.8 \%$ & 0.05 \\
\hline Neck/shoulder symptoms & $52.17 \%(69)$ & $44.69 \%$ & 0.26 \\
\hline Diabetes & $1.5 \%(69)$ & $2.2 \%$ & 0.69 \\
\hline Rheumatoid arthritis & $1.5 \%(68)$ & $1.9 \%$ & 0.82 \\
\hline Retire & $11.6 \%(69)$ & $10 \%$ & 0.69 \\
\hline Job change & $68.1 \%(69)$ & $58.9 \%$ & 0.16 \\
\hline Exercise & $67.5 \%(40)$ & $57.5 \%$ & 0.25 \\
\hline Smoke & $43.5 \%(69)$ & $37.8 \%$ & 0.38 \\
\hline Elbow/forearm worst discomfort rating & $2.65(23)$ & $1.03(70)$ & 0.00 \\
\hline Elbow/forearm worst discomfort rating in last 30 days & $4.34(20)$ & $3.51(49)$ & 0.24 \\
\hline Skill discretion & $24.3(67)$ & $25.5(310)$ & 0.26 \\
\hline Decision authority & $24.5(68)$ & $26.1(315)$ & 0.18 \\
\hline Created & $6.7(67)$ & $6.9(315)$ & 0.62 \\
\hline Coworker support & $11.7(52)$ & $11.6(222)$ & 0.86 \\
\hline Supervisor support & $11.3(67)$ & $11.4(300)$ & 0.72 \\
\hline Job insecurity & $5.3(53)$ & $5.1(228)$ & 0.56 \\
\hline Job satisfaction & $0.35(69)$ & $0.3(312)$ & 0.09 \\
\hline Perceived stress & $24.3(52)$ & $23(227)$ & 0.33 \\
\hline Clerical \% & $66.7 \%(69)$ & $61.3 \%(320)$ & 0.40 \\
\hline Median mononeuropathy $\geq 0.5 \mathrm{~ms}$ (slow $5 \mathrm{~b}$ ) & $23.2 \%(69)$ & $21.3 \%(320)$ & 0.72 \\
\hline Abnormal hand diagram & $30.4 \%(69)$ & $23.8 \%(320)$ & 0.24 \\
\hline Abnormal hand activity threshold limit value (TLV) & $36.1 \%(36)$ & $40.3 \%(181)$ & 0.64 \\
\hline Median ulnar peak latency difference, (dominant side) & $0.26(68)$ & $0.23(320)$ & 0.56 \\
\hline Peak force-dominant side & $2.9(68)$ & $2.9(312)$ & 0.97 \\
\hline New CTS at T2* or in-between & $8.7 \%$ & $8.4 \%$ & 0.90 \\
\hline No CTS ever & $71 \%$ & $83.4 \%$ & 0.02 \\
\hline New shoulder tendonitis at $\mathrm{T} 2$ or in-between & $18.84 \%$ & $8.44 \%$ & 0.01 \\
\hline New wrist/hand/finger tendonitis at $\mathrm{T} 2$ or in-between & $78.26 \%$ & $2.81 \%$ & 0.00 \\
\hline Shoulder tendonitis at $\mathrm{T} 1^{* *}$ & $4.35 \%(69)$ & $4.69 \%(320)$ & 0.90 \\
\hline Wrist/hand/finger tendonitis at $\mathrm{T} 1$ & $7.25 \%(69)$ & $3.75 \%(320)$ & 0.20 \\
\hline CTS at T1 & $11.59 \%(69)$ & $5 \%(320)$ & 0.04 \\
\hline
\end{tabular}

*Time two (follow-up); ${ }^{* *}$ T1-Time one-(baseline). 
Table III. Comparison of 'Incident Cases of Shoulder Tendonitis' to 'No Shoulder Tendonitis'

\begin{tabular}{|c|c|c|c|}
\hline & $\begin{array}{l}\text { Incident cases of } \\
\text { shoulder tendonitis (n) }\end{array}$ & $\begin{array}{c}\text { No shoulder } \\
\text { tendonitis ever (n) }\end{array}$ & $p$ \\
\hline BMI & $29.38(43)$ & $28(339)$ & 0.18 \\
\hline Age & $39.9(43)$ & $38.2(345)$ & 0.31 \\
\hline Gender (female \%) & $72 \%(43)$ & $70 \%(345)$ & 0.71 \\
\hline Repetition & $5.6(43)$ & $5.6(337)$ & 0.96 \\
\hline Wrist/hand/finger symptoms & $60 \%(43)$ & $47 \%(345)$ & 0.11 \\
\hline Elbow/forearm symptoms & $37.2 \%(43)$ & $24.35 \%(345)$ & 0.07 \\
\hline Diabetes & $2.3 \%(43)$ & $2.0 \%(345)$ & 0.89 \\
\hline Rheumatoid arthritis & $2.3 \%(43)$ & $1.7 \%(344)$ & 0.79 \\
\hline Retire & $9.3 \%(43)$ & $11 \%(345)$ & 0.73 \\
\hline Job change & $62.8 \%(43)$ & $60.2 \%(344)$ & 0.74 \\
\hline Exercise & $55 \%(20)$ & $56.9 \%(197)$ & 0.87 \\
\hline Smoke & $30.2 \%(43)$ & $39.7 \%(345)$ & 0.23 \\
\hline Neck/shoulder worst discomfort rating & $2.78(30)$ & $2.18(138)$ & 0.16 \\
\hline Neck/shoulder worst discomfort in last 30 days & $3.17(28)$ & $3.4(113)$ & 0.73 \\
\hline Skill discretion & $24(43)$ & $26(333)$ & 0.18 \\
\hline Decision authority & $24(43)$ & $26.4(339)$ & 0.13 \\
\hline Created & $6.4(43)$ & $7(338)$ & 0.15 \\
\hline Coworker support & $11(28)$ & $12(243)$ & 0.02 \\
\hline Supervisor support & $10(40)$ & $11.5(323)$ & 0.00 \\
\hline Job insecurity & $5(30)$ & $5(248)$ & 0.53 \\
\hline Job satisfaction & $0.35(43)$ & $0.3(336)$ & 0.09 \\
\hline Perceived stress & $24(30)$ & $23(247)$ & 0.74 \\
\hline Clerical \% & $62.8 \%(43)$ & $60.3 \%(345)$ & 0.75 \\
\hline Median mononeuropathy $\geq 0.5 \mathrm{~ms}$ (slow $5 \mathrm{~b}$ ) & $25.6 \%(43)$ & $20.6 \%(345)$ & 0.45 \\
\hline Abnormal hand diagram & $34.9 \%(43)$ & $22.3 \%(345)$ & 0.07 \\
\hline Abnormal hand activity threshold limit value (TLV) & $42.3 \%(26)$ & $40.6 \%(197)$ & 0.87 \\
\hline Median ulnar peak latency difference (dominant side) & $0.16(43)$ & $0.23(344)$ & 0.34 \\
\hline Peak force (dominant side) & $2.96(43)$ & $2.96(336)$ & 0.98 \\
\hline CTS at T2 or in-between & $6.98 \%(43)$ & $8.7 \%(345)$ & 0.70 \\
\hline No CTS ever & $79.07 \%(43)$ & $81.7 \%(345)$ & 0.67 \\
\hline Elbow/forearm tendonitis at $\mathrm{T} 2$ or in-between & $30.23 \%$ & $15.36 \%$ & 0.01 \\
\hline Wrist/Hand/Fingers tendonitis at T2 or in-between & $34.9 \%$ & $15.36 \%$ & 0.00 \\
\hline Wrist/hand/finger tendonitis at $\mathrm{T} 1$ & $6.98 \%(43)$ & $3.77 \%(345)$ & 0.32 \\
\hline Elbow/forearm tendonitis at $\mathrm{T} 1^{* *}$ & $6.98 \%(43)$ & $4.06 \%(345)$ & 0.38 \\
\hline $\mathrm{CTS}$ at $\mathrm{T} 1$ & $6.98 \%(43)$ & $5.22 \%(345)$ & 0.63 \\
\hline CTS at T1 self-report & $9.3 \%$ & $5.51 \%$ & 0.32 \\
\hline CTS + CTSMM5HD & $13.9 \%$ & $9.3 \%$ & 0.33 \\
\hline Shoulder discomfort rating $\geq 5$ & $23 \%(30)$ & $11 \%(138)$ & 0.07 \\
\hline
\end{tabular}

*T2-Time two (follow-up); **T1-Time one-(baseline).

or elbow discomfort at baseline as well as CTS at the baseline evaluation or a history of a prior diagnosis of CTS. There was a trend toward a higher BMI among incident cases. The shoulder tendonitis analysis included 388 workers of which there were 43 incident cases. Among incident shoulder tendonitis cases, the only variables that were significant in the univariate analysis were coworker and supervisor support variables. There was a trend toward incident cases having higher elbow and shoulder discomfort at baseline.

The logistic regression model was created for the combined UET cohort in order to maximize the number of incident cases. The models were run for each regional tendonitis and these findings were similar to the model for the combined UET. The final logistic model determined that several ergonomic factors, age, BMI, history of CTS as well as initial discomfort ratings were significant predictors of incident cases of UET when controlling for the other independent variables. Paradoxically, a higher hand repetition rating was loosely 
Table IV. Logistic Regression Model for Incident Cases of Any Upper Extremity Tendonitis, Odds Ratio and 95\% CI

\begin{tabular}{lccc}
\hline \multicolumn{1}{c}{ Variable } & Odds Ratio & $p$ value & $95 \%$ CI \\
\hline Age $>40$ & 1.76 & 0.03 & $1.04,2.98$ \\
BMI $>$ 30 & 1.93 & 0.02 & $1.12,3.34$ \\
Shoulder discomfort at baseline & 1.84 & 0.04 & $1.03,3.29$ \\
History of CTS & 2.21 & 0.06 & $0.97,5.00$ \\
Worst discomfort rating at baseline & 1.21 & 0.01 & $1.06,1.38$ \\
Shoulder posture rating average & 1.92 & 0.01 & $1.14,3.24$ \\
Hand repetition level & 0.81 & 0.07 & $0.65,1.01$ \\
\hline
\end{tabular}

Note. $N=344 ; p$ value for model $<.001$; Pseudo $R^{2}=0.12$.

associated with a lower risk of UET in the logistic model though this relationship was not found in the univariate analysis; this was a trend and did not reach statistical significance. The hand repetition rating for incident cases and controls was 5.6 in each group at the baseline assessment. The odds ratio and 95\% confidence interval (CI) are presented in Table IV. A worker over the age of 40 was $76 \%$ more likely to develop a UET and a person with a BMI over 30 was almost twice as likely to be an incident case. A baseline complaint of neck or shoulder discomfort increased the risk of becoming an incident case by $84 \%$ and there was an increase of $21 \%$ for every 1 point increase in the baseline discomfort rating. There was a $92 \%$ increased risk for an incident case for each point on the average shoulder posture rating. A worker with a history of CTS or diagnosed as having CTS at the initial screening was more than twice as likely to become an incident case.

Additional logistic regression models were explored using a subset of only active workers and another with only incident cases determined by self report. Neither of these models differed significantly from the results reported for the entire model.

\section{DISCUSSION}

The cumulative incidence rate of developing an UET within the cohort studied was $24.3 \%$ or an estimated $4.5 \%$ annually. This figure is significantly higher than the $1-1.7 \%$ annual rate reported by the BLS for some high risk occupations (3). However, Gerr et al. (5), demonstrated an incidence rate of $21 \%$ among newly hired computer users and Kurppa et al. (4) reported an incidence rate of $21 \%$ among women in a meat packing plant. Our cohort included a variety of occupations including several industrial and clerical sites. Incident cases in our study were diagnosed based, in part, upon a self reporting that a physician had established the diagnosis. We did confirm a diagnosis of UET in approximately half of the workers identified. Gerr et al. (5) did more frequent symptom surveys and followed with physical examinations for all symptomatic workers thus increasing the yield of identifying incident cases (5) The Kurppa study allowed for counting multiple episodes for the same individual if there was an intervening 2 months with medical consultation (4). Of the original study cohort, only $52 \%$ were included in the follow-up. If we assume that none of the subjects lost to follow-up developed tendonitis, we would still have an incident rate of $2.2 \%$, which is relatively high compared to most other published studies.

The factors found to have the highest predictive value for identifying a person who is likely to develop an UET in the near future included age over 40, a BMI over 30, a complaint 
at baseline of a shoulder or neck discomfort, a history of CTS and a job with a higher shoulder posture rating. Age has been identified as a risk factor for UET by other investigators. It is unusual for a person to develop a non-traumatic shoulder tendonitis before the age of 40 but relatively common in older individuals (16). The association between aging and risk for tendonitis is mostly related to the repeated injury over time and the body's decreased ability to repair the injury in a timely fashion. Obesity has not been linked directly to tendonitis in the past. Obesity has been associated with CTS and low back pain in several studies. Recently CTS and UET have been shown to be associated so it is possible that the association is linked to the common relationship with CTS. There is no clear mechanism that explains why increased BMI is a risk factor for UET except the possibility that it acts as an indicator of deconditioning.

A complaint of shoulder discomfort is predictive of a future UET problem. This seems intuitive since these workers may be in the early stages of developing tendonitis and offers an excellent opportunity for a secondary prevention strategy. If any type of symptom screening is done in the workplace, those workers with regional shoulder complaints can be chosen for a focused intervention which could include ergonomic risk assessment, physical therapy or education.

Workers with a history of CTS are more likely to develop an UET. The finding that a history of one area of regional discomfort is predictive of a different musculoskeletal disorder has been demonstrated in two other studies. Ferry et al. (17) reported that women who had a prior history of a musculoskeletal problem were 2.3 times more likely to be diagnosed with CTS. Macfarlane et al. (18) found that individuals in the general population who have a history of a regional pain syndrome are twice as likely to report a new episode of forearm pain. A secondary prevention strategy could be developed once these workers are identified with CTS.

Awkward shoulder postures are also associated with the development of UET. It is well established that any shoulder posture above the chest will put a significant strain on the rotator cuff muscles. Awkward shoulder postures are also linked to wrist and elbow tendonitis in this study cohort. This suggests that the shoulder posture may influence the kinetic chain and exert an influence in the development of muscle strain distal to the shoulder.

The finding of a trend for higher hand repetition to be protective of UET was surprising and does not agree with the findings of other epidemiologic studies. In the univariate analysis the hand repetition level was a neutral factor, not associated with an increased or decreased rate of UET but there is apparently some complex interaction in the multivariate analysis which we have not been able to identify.

\section{CONCLUSION}

Workers with a history of shoulder complaints and awkward shoulder postures are at higher risk for an UET. Older and obese workers and those with a history of CTS are also at higher risk for developing an UET. These risk factors are not highly predictive of an individual's health outcome but do add to our understanding of the natural history of UET among active workers. The risk profile identifies both ergonomic and personal health factors as risks and both types of factors may be amenable to prevention strategies. One strategy for prevention programs would be to focus the resources on those at highest 
risk. This study provides a starting point. Older, heavier workers with a history of upper extremity discomfort or CTS are at the highest risk for developing a new UET. A corporate health promotion that provided an upper extremity-conditioning program might be useful in this population at risk. Additionally, placing these workers at jobs with lower ergonomic stress may be appropriate. This study supports an active surveillance program since this would allow a company to identify the 'at risk' population more clearly and allow them to focus their interventions. Education regarding the early signs and symptoms of tendonitis may be helpful as well since this would allow early treatment which is more likely to be successful.

\section{ACKNOWLEDGMENT}

Support for this research is provided by the National Institute on Disability and Rehabilitation Research of the United States Department of Education, Grant No. H133E980007, "Rehabilitation Engineering Research Center." The opinions contained in this publication are those of the grantee and do not necessarily reflect those of the United States Department of Education.

\section{REFERENCES}

1. Franklin GM, Haug J, Heyer N, Checkoway H, Peck N. Occupational carpal tunnel syndrome in Washington State, 1984-1988. Am J Public Health 1991; 82: 741-746.

2. Hanrahan LP, Higgins D, Anderson H, Haskins L, Tai S. Project SENSOR: Wisconsin surveillance of occupational carpal tunnel syndrome. Wis Med J 1991; 90: 80, 82-83.

3. Bernard BP, ed. Musculoskeletal disorders and workplace factors: A critical review of epidemiologic evidence for work-related musculoskeletal disorders of the neck, upper extremity and low back. 1997. U.S. Department of Health and Human Services, NIOSH publication no. 97-141.

4. Kurppa K, Viikari-Juntura E, et al. Incidence of tenosynovitis or peritendonitis and epicodylitis in a meatprocessing factory. Scand J Work Environ Health. 1991; 17: 32-37.

5. Gerr F, Marcus M, Ensor C, Kleinbaum D, Cohen S, Edwards A, Gentry E, Ortiz DJ, Monteilh C. A prospective study of computer users: I. Study design and incidence of musculoskeletal symptoms and disorders. Am J Ind Med 2002; 41(4): 221-235.

6. Roto P, Kivi P. Prevalence of epicondylitis and tenosynovitis among meatcutters. Scand J Work Environ Health 1984; 10(3): 203-205.

7. McCormack RR Jr, Inman RD, Wells A, Berntsen C, Imbus HR. Prevalence of tendonitis and related disorders of the upper extremity in a manufacturing workforce. J Rheumatol 1990; 17: 958-964.

8. Herberts P, Kadefors R, Andersson G, Petersen I. Shoulder pain in industry: An epidemiological study on welders. Acta Orthop Scand 1981; 52: 299-306.

9. Werner RA, Armstrong TJ. Carpal tunnel syndrome: Ergonomic risk factors and intra carpal canal pressure, carpal tunnel syndrome, physical medicine and rehabilitation clinics of North America 1997; 8(3): 555569 .

10. Kimura J. Electrodiagnosis in diseases of nerve and muscle: Principles and practice. Philadelphia: FA Davis, 1983.

11. Latko WA, Armstrong TJ, Foulke JA, Herrin GD, Rabourn RA, Ulin SS. Development and evaluation of an observational method for assessing repetition in hand tasks. Am Ind Hyg Assoc J 1997; 58(4): 278 285.

12. ACGIH Worldwide. Threshold limit values for chemical substances and physical agents in the work environment, 2002.

13. Karasek R, Brisson C, Kawakami N, Houtman I, Bongers P, Amick B. The Job Content Questionnaire (JCQ): An instrument for internationally comparative assessments of psychosocial job characteristics. J Occup Health Psych 1998; 3(4): 322-355.

14. Werner RA, Hamann C, Franzblau A, Rodgers P. Prevalence of carpal tunnel syndrome and upper extremity tendonitis among dental hygienists. J Dent Hygiene 2002; 76(2): 126-132. 
15. Stevens JC, Beard CM, O'Fallon WM, Kurland LT. Conditiona associated with carpal tunnel syndrome. Mayo Clin Proc 1992; 67: 541-548.

16. Snider RK, ed. Essentials of musculoskeletal care 1997, American Academy of Orthopaedic Surgeons, Rosemont, IL, pp. 114-119.

17. Ferry S, Hannaford P, Warskyj M, Lewis M, Croft P. Carpal tunnel syndrome: A nested case control study of risk factors in women. Am J Epidemiol 2000; 151: 566-74.

18. Macfarlane GJ, Hunt IM, Silman AJ. Role of mechanical and psychosocial factors in the onset of forearm pain: Prospective population based study. BMJ 2000; 321: 676-679. 